\title{
Axion emission from supernova with axion-pion-nucleon contact interaction
}

\author{
Kiwoon Choi, ${ }^{a}$ Hee Jung Kim, ${ }^{a}$ Hyeonseok Seong ${ }^{a}$ and Chang Sub Shin ${ }^{b, a}$ \\ ${ }^{a}$ Center for Theoretical Physics of the Universe, Institute for Basic Science (IBS), \\ 34126 Daejeon, South Korea \\ ${ }^{b}$ Department of Physics and Institute of Quantum Systems (IQS), \\ Chungnam National University, 34134 Daejeon, South Korea \\ E-mail: kchoi@ibs.re.kr, heejungkim@ibs.re.kr, hseong@ibs.re.kr, \\ csshin@cnu.ac.kr
}

ABSTRACT: We examine the axion emission from supernovae with a complete set of relevant axion couplings including the axion-pion-nucleon contact interaction which was ignored in the previous studies. Two processes are affected by the axion-pion-nucleon contact interaction, $\pi^{-}+p \rightarrow n+a$ and $n+p \rightarrow n+p+a$, and these processes can be the dominant source of axions for some region in the axion parameter space or in astrophysical conditions encountered inside supernovae. We find that the contact interaction can enhance the axion emissivity of $\pi^{-}+p \rightarrow n+a$ by a factor of $2-4$, while the effect on $n+p \rightarrow n+p+a$ is not significant. We also discuss the relative importance of other pion-induced processes such as $\pi^{0}+n \rightarrow n+a$ and $\pi^{-}+\pi^{0} \rightarrow \pi^{-}+a$.

Keywords: Beyond Standard Model, Cosmology of Theories beyond the SM

ArXiv EPrint: 2110.01972 


\section{Contents}

1 Introduction 1

2 Axion couplings to nucleons and pions 3

3 Axion emission from supernovae by hadronic processes $\quad 6$

$\begin{array}{lll}3.1 & \text { Pion-nucleon scattering } & 6\end{array}$

$\begin{array}{lll}3.2 & \text { Nucleon-nucleon bremsstrahlung } & 10\end{array}$

$\begin{array}{lll}3.3 & \text { Pion-pion scattering: } \pi+\pi \rightarrow \pi+a & 14\end{array}$

4 Conclusions and discussion $\quad 15$

A Axion emissivity from $n+p \rightarrow n+p+a$ in degenerate limit $\quad 16$

\section{Introduction}

The axion which was initially introduced as a solution to the strong CP problem [1-4] has turned out to have many interesting phenomenological consequences [5-7]. After recognizing that axions provide a compelling candidate for the dark matter in the Universe [8-10], a lot of efforts have been made to search for axions over the parameter space of the representative axion models [11-14]. Since the viable parameter region is in the very weakly coupled regime, most of the laboratory experiments searching for axions are ongoing in the direction of the precision measurements using, for example, resonant cavities, nuclear magnetic resonance, light shining through the walls, and polarization of lights in magnetic fields (see [15-17] for comprehensive reviews).

A complementary approach which can severely constrain the couplings of light axions is to use astrophysical objects forming a hot and dense environment, e.g., supernovae, stars on the horizontal and red giant branches, neutron stars, and even white dwarfs (see [18] for a review and also [19] for a recent overview). Axions can be produced abundantly from those objects, thereby altering their evolution. One can then derive constraints on the couplings of axions by requiring that the axion emission does not significantly alter the standard evolution scenario which is consistent with the observational data.

A core-collapse supernova, e.g., SN1987A, is known to provide stringent constraints on the axion couplings to hadrons, particularly on the couplings to nucleons [20, 21]. The observation of the neutrino flux from SN1987A, which is consistent with the standard scenario [22, 23], suggests that the additional cooling by axion emission from the associated proto-neutron star is constrained as $L_{a} \lesssim L_{\nu}=\mathcal{O}(1-10) \times 10^{51} \mathrm{erg} / \mathrm{sec}$, where $L_{a}$ and $L_{\nu}$ denote the axion and neutrino luminosities around $1-10 \mathrm{sec}$ after the formation of the proto-neutron star [18].

Among the processes producing axions from supernovae, the nucleon bremsstrahlung process $N+N \rightarrow N+N+a(N=n, p)$ has been considered as the dominating process 
for many years [24-28]. However, recently it has been noticed that the number density of negatively charged pions inside supernovae can be significantly enhanced by pion-nucleon interactions [29]. Based on this observation, the pion-induced Compton-like process $\pi^{-}+$ $p \rightarrow n+a$, which was originally studied in [30,31], has been revisited. Taking into account medium effects, refs. [32, 33] show that the process dominates over the nucleon-nucleon bremsstrahlung for a wide range of astrophysical conditions encountered inside supernovae. ${ }^{1}$

Motivated by the importance of the process $\pi^{-}+p \rightarrow n+a$, in this paper we extend the previous analysis of axion emission from supernovae with a complete set of relevant axion couplings including the axion-pion-nucleon and axion-pion contact interactions which were ignored in the previous studies. Our primary concern is how significantly the contact interactions can affect the axion emissivity. We start with a general axion Lagrangian above the QCD confinement scale, which determines the axion couplings to hadrons below that scale. To highlight the coupling dependence of the axion emissivity more clearly, we compare a new contribution including the effect of contact terms to that from the axionnucleon couplings only and take the ratio between the two contributions. It is expected that the ratios can lead to cancellation of the uncertainties in nuclear physics and the medium effect. Thus, as a first step towards understanding the contributions of the contact interactions, we consider the tree-level diagrams in the leading order pion-nucleon couplings and the one-pion exchange diagrams for the nucleon-nucleon bremsstrahlung. We also ignore the background matter effect, which should be included in future work. In such an approximation, two processes are affected by the axion-pion-nucleon contact interaction, $\pi^{-}+p \rightarrow n+a$ and $n+p \rightarrow n+p+a$. We find that the axion-pion-nucleon contact interaction can enlarge the emission rate of $\pi^{-}+p \rightarrow n+a$ by a factor of $2-4$ depending on the pattern of axion couplings, while the effect on $n+p \rightarrow n+p+a$ is negligible. We also examine other pion-induced processes such as $\pi^{0}+n \rightarrow n+a$ and $\pi^{-}+\pi^{0} \rightarrow \pi^{-}+a$, where the latter process is induced by the axion-pion contact interaction. We then find that $\pi^{0}+n \rightarrow n+a$ can be as important as $\pi^{-}+p \rightarrow n+a$, again depending on the pattern of axion couplings, while $\pi^{-}+\pi^{0} \rightarrow \pi^{-}+a$ is negligible compared to $\pi^{-}+p \rightarrow n+a$ over the entire axion parameter space for astrophysical conditions encountered inside proto-neutron stars.

This paper is organized as follows. In section 2, we introduce the relevant axion couplings to nucleons and pions in the context of a generic axion model and discuss the model dependence of couplings for a simple class of axion models. In section 3, we investigate the axion emission from supernovae by a variety of pion-induced processes and the nucleon-nucleon bremsstrahlung processes, with a complete set of relevant axion couplings. Section 4 is a summary and conclusion.

\footnotetext{
${ }^{1}$ The medium effects also modify the axion-nucleon couplings. The modification is expected to be an $\mathrm{O}(1)$ effect in general, while it could result in $\sim 10$-times enhancement of the axion-neutron coupling in the KSVZ model because the accidental cancellation of that coupling in vacuum is spoiled [34]. Here we presume the values of axion couplings in vacuum for our numerical estimation.
} 


\section{Axion couplings to nucleons and pions}

In this section, we briefly discuss the axion couplings to nucleons and pions for generic axions whose couplings are constrained only by the (approximate) global U(1) PecceiQuinn (PQ) symmetry [1-4]. Without loss of generality, at scales below the axion decay constant $f_{a}$, one can always choose a field basis for which only the axion field transforms under the PQ symmetry as

$$
\mathrm{U}(1)_{\mathrm{PQ}}: \quad a \rightarrow a+\text { constant }
$$

while all other fields are invariant [35]. In such a field basis, the axion couplings at low energy scales around $\mu=\mathcal{O}(1) \mathrm{GeV}$ include

$$
\mathcal{L}_{\mathrm{eff}}=c_{G} \frac{g_{s}^{2}}{32 \pi^{2}} \frac{a}{f_{a}} G_{\mu \nu}^{a} \tilde{G}^{a \mu \nu}+\frac{\partial_{\mu} a}{2 f_{a}}\left(C_{u} \bar{u} \gamma^{\mu} \gamma_{5} u+C_{d} \bar{d} \gamma^{\mu} \gamma_{5} d\right)
$$

where the axion decay constant $f_{a}$ defines the axion field range as $a \cong a+2 \pi f_{a}, G_{\mu \nu}^{a}$ are the gluon field strength, and $u$ and $d$ are the up and down quarks. Here $c_{G}$ is an integervalued parameter describing the $\mathrm{U}(1)_{\mathrm{PQ}}$ breaking by the $\mathrm{QCD}$ anomaly, while $C_{u}$ and $C_{d}$ are continuous real-valued parameters describing the $\mathrm{U}(1)_{\mathrm{PQ}}$-preserving axion couplings to the light quarks renormalized at $\mu=\mathcal{O}(1) \mathrm{GeV}$.

For axion models which have a UV completion with a linearly realized $\mathrm{U}(1)_{\mathrm{PQ}}$, the low energy parameters $c_{G}$ and $C_{u, d}$ in eq. (2.2) are determined mainly by the $\mathrm{U}(1)_{\mathrm{PQ}}$ charges defined in the UV model. ${ }^{2}$ As an illustrative example, let us consider axion models in which the first generation quark masses are generated by the following Yukawa couplings: ${ }^{3}$

$$
\mathcal{L}_{\text {Yukawa }}=\lambda_{u}\left(\frac{\sigma}{\Lambda}\right)^{n_{u}} Q_{1} u_{1}^{c} H_{u}+\lambda_{d}\left(\frac{\sigma}{\Lambda}\right)^{n_{d}} Q_{1} d_{1}^{c} H_{d}+\text { h.c. }
$$

where $\sigma$ is a PQ-charged gauge-singlet scalar field whose vacuum expectation value determines the axion decay constant as

$$
\langle\sigma\rangle=\frac{1}{\sqrt{2}} f_{a} e^{i a / f_{a}},
$$

$Q_{i}$ and $u_{i}^{c}, d_{i}^{c}(i=1,2,3)$ denote the three generations of the left-handed $\mathrm{SU}(2)_{L^{-}}$-doublet quarks and the left-handed $\mathrm{SU}(2)_{L^{-}}$-singlet antiquarks, respectively, $H_{u}$ and $H_{d}$ are $\mathrm{SU}(2)_{L^{-}}$ doublet Higgs fields, and finally $\Lambda$ is a cutoff scale of the model. To derive the low energy axion couplings in this model, we first make the following axion-dependent field redefinition at a scale around $f_{a}$ :

$$
\Phi \rightarrow e^{i q_{\Phi} a / f_{a}} \Phi \quad\left(\Phi=\psi, H_{u, d}\right),
$$

and subsequently integrate out all massive fields heavier than $\mu=\mathcal{O}(1) \mathrm{GeV}$, where $q_{\Phi}$ is the PQ charge of $\Phi$ (in the normalization convention with $q_{\sigma}=1$ ) for the linearly

\footnotetext{
${ }^{2}$ For string-theoretic axions that arise from the zero modes of higher-dimensional $p$-form gauge field, there is no UV completion with a linearly realized $\mathrm{U}(1)_{\mathrm{PQ}}$. It has been noted that the tree-level values of $C_{u, d}$ for string-theoretic axions are of the order of $\alpha_{\mathrm{GUT}} / 2 \pi[36]$.

${ }^{3}$ Here for simplicity we ignore the effects of flavor mixings.
} 
realized $\mathrm{U}(1)_{\mathrm{PQ}}$, and $\psi$ stands for all chiral fermions in the model. Then the axion-gluon coupling $c_{G}$, which arises as a consequence of the axion-dependent field redefinition of $\psi$, corresponds to the coefficient of the $\mathrm{U}(1)_{\mathrm{PQ}}-\mathrm{SU}(3)_{c}-\mathrm{SU}(3)_{c}$ anomaly, while the couplings $C_{u, d}$ to the light quarks are determined by (i) a contribution from the axion-dependent fieldredefinition of $\left\{Q_{1}, u_{1}^{c}, d_{1}^{c}\right\}$, (ii) the tree-level threshold correction from the axion mixing with the $Z$ boson which is induced by the field redefinition of $H_{u, d}$, and finally (iii) the radiative corrections caused by the gauge and Yukawa couplings in the model [36]. Putting these together, one finds

$$
\begin{aligned}
c_{G} & =2 \sum_{\psi} q_{\psi} \operatorname{Tr}\left(T_{c}^{2}(\psi)\right), \\
C_{u} & =-n_{u}-\left(q_{H_{u}}+q_{H_{d}}\right) \cos ^{2} \beta+\Delta C_{u}, \\
C_{d} & =-n_{d}-\left(q_{H_{u}}+q_{H_{d}}\right) \sin ^{2} \beta+\Delta C_{d},
\end{aligned}
$$

where $T_{c}(\psi)$ is the color charge of $\psi, \tan \beta=\left\langle H_{u}\right\rangle /\left\langle H_{d}\right\rangle$, and the radiative corrections $\Delta C_{u, d}=\mathcal{O}\left(10^{-2}-10^{-3}\right)$ can be safely ignored if the tree level values of $C_{u, d}$ are of order unity [36].

The above results indicate that a variety of different patterns of $c_{G}$ and $C_{u, d}$ are possible even within the framework of relatively simple axion models. Let us present explicitly the parameter values for some examples. In the $\mathrm{KSVZ}$ model $[11,12], H_{u}=\left(i \sigma_{2} H_{d}\right)^{*}$ and all SM fields are neutral under the linearly realized $\mathrm{U}(1)_{\mathrm{PQ}}$, and therefore $n_{u}=n_{d}=$ $q_{H_{u}}=q_{H_{d}}=0$. The model also involves a heavy $\mathrm{PQ}$-charged exotic quark $\mathcal{Q}$ generating the $\mathrm{U}(1)_{\mathrm{PQ}}-\mathrm{SU}(3)_{c}-\mathrm{SU}(3)_{c}$ with $c_{G}=1$. The resulting couplings of the KSVZ axion at $\mu=\mathcal{O}(1) \mathrm{GeV}$ are given by

$$
\mathrm{KSVZ}: \quad c_{G}=1, \quad C_{u}=\Delta C_{u}=\mathcal{O}\left(10^{-2}\right), \quad C_{d}=\Delta C_{d}=\mathcal{O}\left(10^{-2}\right),
$$

where $\Delta C_{u, d}$ are induced mostly by the axion-gluon coupling $c_{G}$ causing a running of $C_{u, d}$ over the scales from the mass of the exotic quark $\mathcal{Q}$ to $\mu=\mathcal{O}(1) \mathrm{GeV}$ [36]. On the other hand, the minimal DFSZ model $[13,14]$ has $n_{u}=n_{d}=0, q_{H_{u}}=q_{H_{d}}=-1$ and all chiral fermions in the SM model have $q_{\psi}=1 / 2$, which result in

$$
\text { DFSZ : } \quad c_{G}=6, \quad C_{u}=2 \cos ^{2} \beta+\Delta C_{u}, \quad C_{d}=2 \sin ^{2} \beta+\Delta C_{d}
$$

with $\Delta C_{u, d}=\mathcal{O}\left(10^{-3}\right)$ which are smaller than those of the KSVZ model because in the DFSZ model the running of $C_{u, d}$ starts from a lower scale around the top quark mass [36]. It is an interesting possibility that $\mathrm{U}(1)_{\mathrm{PQ}}$ plays the role of a flavor symmetry which explains the fermion mass hierarchies [37-39]. In such a case, $n_{u, d}$ can be non-zero integers and the model can have a more diverse pattern of $c_{G}$ and $C_{u, d}$. Note that, while $n_{u, d}$ in the Yukawa couplings (eq. (2.3)) are required to be non-negative, the sign in front of $n_{u, d}$ in eq. (2.6) can be flipped by replacing $\sigma$ in eq. (2.3) with $\sigma^{*}$. One can further generalize the model by introducing additional $\mathrm{U}(1)_{\mathrm{PQ}}$-charged Higgs doublet, and then $C_{u, d}$ receive additional contribution depending on the vacuum expectation value of the added Higgs field. With this observation, in the following we regard $C_{u}$ and $C_{d}$ as real-valued free parameters, 
and $c_{G}$ as an integer-valued additional free parameter, without specifying the underlying UV model.

From the couplings in eq. (2.2) defined at $\mu=\mathcal{O}(1) \mathrm{GeV}$, we can derive the axion couplings to nucleons and pions which are relevant for the axion emission from supernova. Including the conventional pion-nucleon couplings, the interactions are given by $[6,40]$

$$
\begin{aligned}
\mathcal{L}_{\text {int }}= & \frac{g_{A}}{2 f_{\pi}}\left(\partial_{\mu} \pi^{0}\left(\bar{p} \gamma^{\mu} \gamma_{5} p-\bar{n} \gamma^{\mu} \gamma_{5} n\right)+\sqrt{2} \partial_{\mu} \pi^{+} \bar{p} \gamma^{\mu} \gamma_{5} n+\sqrt{2} \partial_{\mu} \pi^{-} \bar{n} \gamma^{\mu} \gamma_{5} p\right) \\
& +\frac{\partial_{\mu} a}{2 f_{a}}\left(C_{a p} \bar{p} \gamma^{\mu} \gamma_{5} p+C_{a n} \bar{n} \gamma^{\mu} \gamma_{5} n+\frac{C_{a \pi N}}{f_{\pi}}\left(i \pi^{+} \bar{p} \gamma^{\mu} n-i \pi^{-} \bar{n} \gamma^{\mu} p\right)\right) \\
& +\frac{\partial_{\mu} a}{2 f_{a}} \frac{C_{a \pi}}{f_{\pi}}\left(\pi^{0} \pi^{+} \partial^{\mu} \pi^{-}+\pi^{0} \pi^{-} \partial^{\mu} \pi^{+}-2 \pi^{+} \pi^{-} \partial^{\mu} \pi^{0}\right),
\end{aligned}
$$

where $f_{\pi}=92.4 \mathrm{MeV}$ is the pion decay constant and

$$
\begin{aligned}
C_{a p}-C_{a n} & =g_{A}\left(C_{u}-C_{d}+\left(\frac{m_{u}-m_{d}}{m_{u}+m_{d}}\right) c_{G}\right), \\
C_{a p}+C_{a n} & =g_{0}\left(C_{u}+C_{d}-c_{G}\right), \\
C_{a \pi N} & =\frac{C_{a p}-C_{a n}}{\sqrt{2} g_{A}}, \quad C_{a \pi}=\frac{2\left(C_{a p}-C_{a n}\right)}{3 g_{A}}
\end{aligned}
$$

with the nucleon matrix elements of the light quark axial vector currents given by

$$
\begin{aligned}
g_{A} & =\Delta u-\Delta d \simeq 1.2723(23), \\
g_{0} & =\Delta u+\Delta d \simeq 0.521(53),
\end{aligned}
$$

where $s^{\mu} \Delta q=\left\langle N\left|\bar{q} \gamma^{\mu} \gamma_{5} q\right| N\right\rangle(q=u, d)$ for the nucleon spin four vector $s^{\mu}$. Here the numerical value of $g_{0}$ is chosen for the axion-quark couplings $C_{u, d}$ renormalized at $\mu=$ $2 \mathrm{GeV}$ in the $\overline{\mathrm{MS}}$ scheme [41], and the small contributions from the axion couplings to the heavier quarks $Q=\{s, c, b, t\}$ are ignored.

The above results show that the entire axion couplings to nucleons and pions, including the axion-pion-nucleon contact interaction $C_{a \pi N}$ and the axion-pion contact interaction $C_{a \pi}$, are determined by the two free parameters $C_{a n}$ and $C_{a p}$. An interesting feature of these parameters is that in some axion models they can have a hierarchical pattern such as $\left|C_{a p}\right| \gg\left|C_{a n}\right|$ or $\left|C_{a p}-C_{a n}\right| \gg\left|C_{a p}+C_{a n}\right|$ without fine tuning of any continuous parameter in the underlying UV model. For instance, including the radiative corrections induced by the axion-gluon coupling $c_{G}$, the KSVZ and string-theoretic axions have $\left|C_{u, d}\right|=$ $\mathcal{O}\left(10^{-2} c_{G}\right)[36]$, which results in

$$
\left|C_{a p}\right| \simeq 0.48\left|c_{G}\right| \gg\left|C_{a n}\right|=\mathcal{O}\left(10^{-2}\left|c_{G}\right|\right)
$$

for the nucleon matrix elements in eq. (2.11) and the light quark mass ratio $m_{u} / m_{d}=$ 0.48(3). Also, for the axion couplings in eq. (2.6), the anomaly coefficient $c_{G}$ and the tree level value of $C_{u}+C_{d}$ are all quantized parameters. Then, for a model with $\mathrm{U}_{(1)_{\mathrm{PQ}^{-}}}$ charges yielding

$$
c_{G}=-\left(n_{u}+n_{d}+q_{H_{u}}+q_{H_{d}}\right)=\mathcal{O}(1),
$$


the model predicts

$$
C_{a p}-C_{a n}=\mathcal{O}(1), \quad C_{a p}+C_{a n}=g_{0}\left(\Delta C_{u}+\Delta C_{d}\right) \lesssim \mathcal{O}\left(10^{-2}\right)
$$

At any rate, axions generically have the axion-pion-nucleon contact interaction given by $C_{a \pi N}=\left(C_{a p}-C_{a n}\right) / \sqrt{2} g_{A}\left(g_{A} \simeq 1.27\right)$ and the axion-pion contact interaction $C_{a \pi}=$ $2\left(C_{a p}-C_{a n}\right) / 3 g_{A}$. On the other hand, these contact interactions were not taken into account in the previous studies of axion emission from supernovae. In section 3, we will examine the effects of those contact interactions on the axion emission rates to see how important they can be.

\section{Axion emission from supernovae by hadronic processes}

In this section, we examine the axion production by hadron collisions inside a newly born proto-neutron star. We consider three types of processes, the pion-nucleon scattering $\pi+$ $N \rightarrow N+a(N=n, p)$, the nucleon-nucleon bremsstrahlung $N+N \rightarrow N+N+a$, and the pion-pion scattering $\pi+\pi \rightarrow \pi+a$. The relative importance of each process depends on the pattern of axion couplings, as well as on the density and temperature of the corresponding astrophysical environment. Our prime goal is to examine the effects of the two contact interactions, the axion-pion-nucleon contact coupling $C_{a \pi N}$ and the axion-pion contact coupling $C_{a \pi}$ in eq. (2.9), which were not taken into account before except for the nucleon-nucleon bremsstrahlung [42]. We will examine this question in a simple approximation keeping only the leading order in pion-nucleon couplings and ignoring medium effects. Accordingly, we can find a simple form of the coupling dependence in that approximation, and it shows the relative importance of the contribution from each coupling at a rough estimate.

\subsection{Pion-nucleon scattering}

Let us first discuss the pion-nucleon scattering process $\pi+N \rightarrow N+a$. For $T \sim 40 \mathrm{MeV}$ and the nucleon mass density $\rho \sim 10^{14} \mathrm{~g} / \mathrm{cm}^{3}$ encountered inside a proto-neutron star, the pion and nucleon number densities roughly obey [29]

$$
\frac{n_{\pi^{0}}}{n_{\pi^{-}}} \sim \frac{n_{\pi^{+}}}{n_{\pi^{0}}} \sim \frac{n_{p}}{n_{n}}=\mathcal{O}(0.1) .
$$

It is then expected that $\pi^{-}+p \rightarrow n+a$ and $\pi^{0}+n \rightarrow n+a$ are the dominating process depending upon the involved axion couplings. The Feynman diagrams for these processes are depicted in figure 1 and figure 2, showing that at leading order in pion-nucleon couplings $\pi^{0}+n \rightarrow n+a$ involves only the axion-neutron coupling $C_{a n}$, while $\pi^{-}+p \rightarrow n+a$ depends on three axion couplings, $C_{a p}, C_{a n}$, and the axion-pion-nucleon contact interaction $C_{a \pi N}$.

Recently, the process $\pi^{-}+p \rightarrow n+a$ has been argued to be the dominating process to produce axions for a wide range of astrophysical conditions encountered inside supernovae $[32,33]$. The axion emissivity (the energy loss induced by axion emissions per unit 

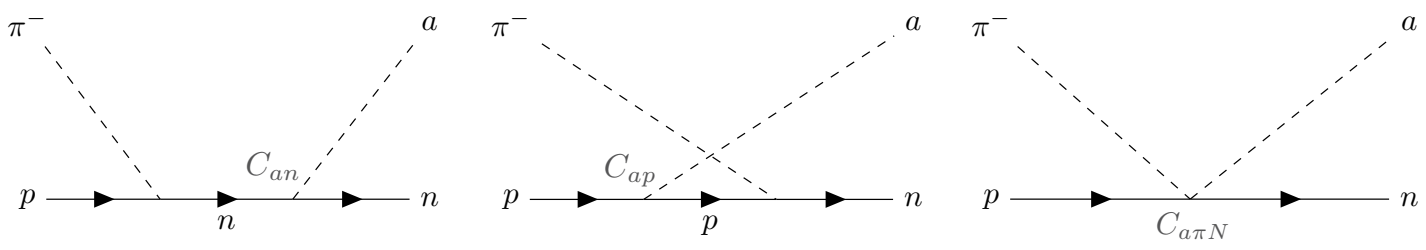

Figure 1. Diagrams for $\pi^{-}+p \rightarrow n+a$ from the axion couplings in eq. (2.9).
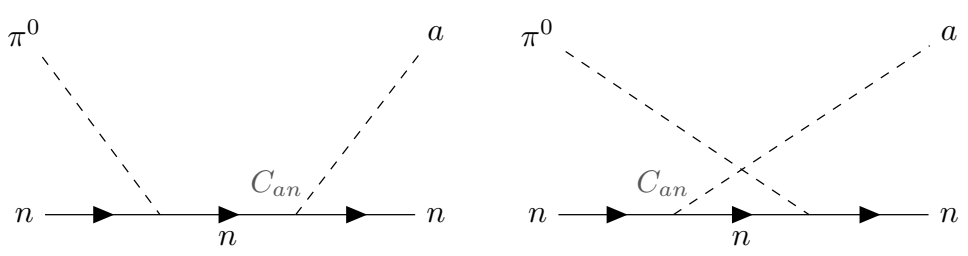

Figure 2. Diagrams of axion production for the process $\pi^{0}+n \rightarrow n+a$.

volume and second) of this process is given by

$$
\begin{aligned}
Q_{a}^{p \pi^{-}}=\int \prod_{\alpha=\pi, p, n, a} & \frac{d^{3} \mathbf{p}_{\alpha}}{(2 \pi)^{3} 2 E_{\alpha}}\left[(2 \pi)^{4} \delta^{(4)}\left(p_{\pi}+p_{p}-p_{n}-p_{a}\right)\right. \\
& \left.\times f_{\pi}\left(p_{\pi}\right) f_{p}\left(p_{p}\right)\left(1-f_{n}\left(p_{n}\right)\right) \sum_{s_{p}, s_{n}}\left|\mathcal{M}_{\pi^{-}+p \rightarrow n+a}\right|^{2} E_{a}\right],
\end{aligned}
$$

where $p_{\alpha}=\left(E_{\alpha}, \mathbf{p}_{\alpha}\right)$ are the particle four-momenta, $f_{\alpha}\left(p_{\alpha}\right)$ are the Fermi-Dirac or BoseEinstein distribution function, and $s_{N}(N=p, n)$ denotes the nucleon spin. Although the integrand has angular dependence after applying the energy-momentum conservation, the nucleon distribution functions can be approximated to be independent of those angles in the non-relativistic limit. Then, the squared matrix element can be first integrated over the relative angle of $\mathbf{p}_{p}$ with respect to $\mathbf{p}_{a}$, while the integration over the solid angle of $\mathbf{p}_{a}$ amounts to a factor of $4 \pi .{ }^{4}$ Taking the non-relativistic limit for the initial proton and integrating over the relative angle between $\mathbf{p}_{\pi^{-}}$and $\mathbf{p}_{a}$, we find

$$
\int d \Omega_{\pi^{-}} \sum_{s_{p}, s_{n}}\left|\mathcal{M}_{\pi^{-}+p \rightarrow n+a}\right|^{2}=\frac{8 \pi m_{N}^{4}}{f_{a}^{2} f_{\pi}^{2}} \mathcal{C}_{a}^{p \pi^{-}},
$$

where $m_{N}$ is the nucleon mass and $\mathcal{C}_{a}^{p \pi^{-}}$is a dimensionless quantity which can be expanded in powers of $1 / m_{N}$ as

$$
\begin{aligned}
\mathcal{C}_{a}^{p \pi^{-}} \simeq & \frac{2}{3} g_{A}^{2}\left(\frac{\left|\mathbf{p}_{\pi}\right|}{m_{N}}\right)^{2}\left(2 C_{+}^{2}+C_{-}^{2}\right)+\left(\frac{E_{\pi}}{m_{N}}\right)^{2} C_{a \pi N}^{2} \\
& +\sqrt{2} g_{A}\left(\frac{E_{\pi}}{m_{N}}\right)^{3}\left(1-\frac{1}{3}\left(\frac{\left|\mathbf{p}_{\pi}\right|}{E_{\pi}}\right)^{2}\right) C_{a \pi N} C_{-},
\end{aligned}
$$

\footnotetext{
${ }^{4}$ The squared matrix element depends on two independent angles, each from the Mandelstam variables $s$ and $u$. We approximate these Mandelstam variables as $s=\left(p_{p}+p_{\pi^{-}}\right)^{2} \simeq m_{p}^{2}+m_{\pi^{-}}^{2}+2 m_{p} E_{\pi^{-}}$and $u=\left(p_{p}-p_{a}\right)^{2} \simeq m_{p}^{2}-2 m_{p} E_{a}$.
} 
where

$$
C_{ \pm}=\frac{1}{2}\left(C_{a p} \pm C_{a n}\right), \quad E_{\pi}=\sqrt{m_{\pi^{-}}^{2}+\left|\mathbf{p}_{\pi}\right|^{2}} .
$$

We remark that the above expression of $\mathcal{C}_{a}^{p \pi^{-}}$corresponds to the leading order result (in $\left.1 / m_{N}\right)$ for which the three axion coupling combinations, i.e., $2 C_{+}^{2}+C_{-}^{2}, C_{a \pi N} C_{-}$and $C_{a \pi N}^{2}$, are pretended to be independent parameters. As already noticed, $C_{a \pi N}$ is not an independent parameter, but is determined as $C_{a \pi N}=\sqrt{2} C_{-} / g_{A}$ (see eq. (2.10)). Then the third term can be interpreted as a higher order term as it is suppressed compared to other terms by additional power of $E_{\pi} / m_{N}$. However, our numerical estimation gives $\mathcal{C}_{a}^{p \pi^{-}} \simeq 0.02\left(2 C_{+}^{2}+C_{-}^{2}\right)+0.04 C_{a \pi N}^{2}+0.01 C_{a \pi N} C_{-}$for typical parameter values, e.g. $T=40 \mathrm{MeV},\left|\mathbf{p}_{\pi}\right| \simeq \sqrt{3 m_{\pi^{-}} T} \simeq 130 \mathrm{MeV}$. Note that the third term is comparable to the others. The relative importance of each term coincides with the final axion emissivity in eq. (3.7) up to a small enhancement by the phase space integration.

For $\left|\mathbf{p}_{p}\right| \gg\left|\mathbf{p}_{\pi, a}\right|$, the axion emissivity can be further approximated as

$$
Q_{a}^{p \pi^{-}} \simeq \frac{z_{p} z_{\pi^{-}}}{f_{a}^{2} f_{\pi}^{2}} \sqrt{\frac{m_{N}^{7} T^{11}}{128 \pi^{10}}} \int d x_{p}\left(\frac{x_{p}^{2} e^{x_{p}^{2}}}{\left(e^{x_{p}^{2}}+z_{n}\right)\left(e^{x_{p}^{2}}+z_{p}\right)}\right) \int d x_{\pi}\left(\frac{x_{\pi}^{2} \epsilon_{\pi} \mathcal{C}_{a}^{p \pi^{-}}}{e^{\epsilon_{\pi}-y_{\pi}}-z_{\pi^{-}}}\right),
$$

where $z_{i}=e^{\left(\mu_{i}-m_{i}\right) / T}$ are the fugacities, $\epsilon_{\pi}=E_{\pi} / T, y_{\pi}=m_{\pi^{-}} / T, x_{\pi}=\left|\mathbf{p}_{\pi}\right| / T$, and $x_{p}=\left|\mathbf{p}_{p}\right| / \sqrt{2 m_{N} T}$. The emissivity eq. (3.6) depends on many astrophysical parameters which are related to each other by the equation of state, e.g., the temperature $T$ and the chemical potentials $\mu_{i}\left(i=n, p, \pi^{-}\right)$. To parameterize the astrophysical condition in terms of $T$ and the total mass density $\rho$, we use the fugacities obtained in [29] and numerically calculate the integral in eq. (3.6) around $T \sim 40 \mathrm{MeV}$ and $\rho \sim 10^{14} \mathrm{~g} / \mathrm{cm}^{3}$. We then find

$$
\begin{aligned}
\frac{Q_{a}^{p \pi^{-}}}{\mathrm{erg} \cdot \mathrm{cm}^{-3} \mathrm{~s}^{-1}} \simeq & 1.4 \times 10^{33} T_{40}^{7.2} \rho_{14}^{1.1}\left(\frac{10^{9} \mathrm{GeV}}{f_{a}}\right)^{2}\left(2 C_{+}^{2}+C_{-}^{2}\right) \\
& +2.3 \times 10^{33} T_{40}^{6.6} \rho_{14}^{1.1}\left(\frac{10^{9} \mathrm{GeV}}{f_{a}}\right)^{2} C_{a \pi N}^{2} \\
& +1.2 \times 10^{33} T_{40}^{7.5} \rho_{14}^{1.1}\left(\frac{10^{9} \mathrm{GeV}}{f_{a}}\right)^{2} C_{a \pi N} C_{-}
\end{aligned}
$$

where $T_{40} \equiv T /(40 \mathrm{MeV})$ and $\rho_{14} \equiv \rho /\left(10^{14} \mathrm{~g} / \mathrm{cm}^{3}\right)$. We stress that the above approximation is valid only for a narrow range of $T$ and $\rho$, i.e., for $T_{40} \in[0.9,1.1]$ and $\rho_{14} \in[1,3]$, which is enough for our purpose to examine the effect of the axion-pion-nucleon contact interaction for an ambient condition inside supernovae. ${ }^{5}$

Obviously the second and third terms in the r.h.s. of eq. (3.7) represent the contributions to the axion emissivity from the axion-pion-nucleon contact interaction $C_{a \pi N}$.

\footnotetext{
${ }^{5}$ The emissivity of $\pi^{-}+p \rightarrow n+a$ obtained in [32] for $C_{a \pi N}=0$ is bigger than ours by a factor $\sim 2$. As the analysis of [32] takes into account leading order medium effects, while ours does not, it is likely that this difference originates from medium effects.
} 

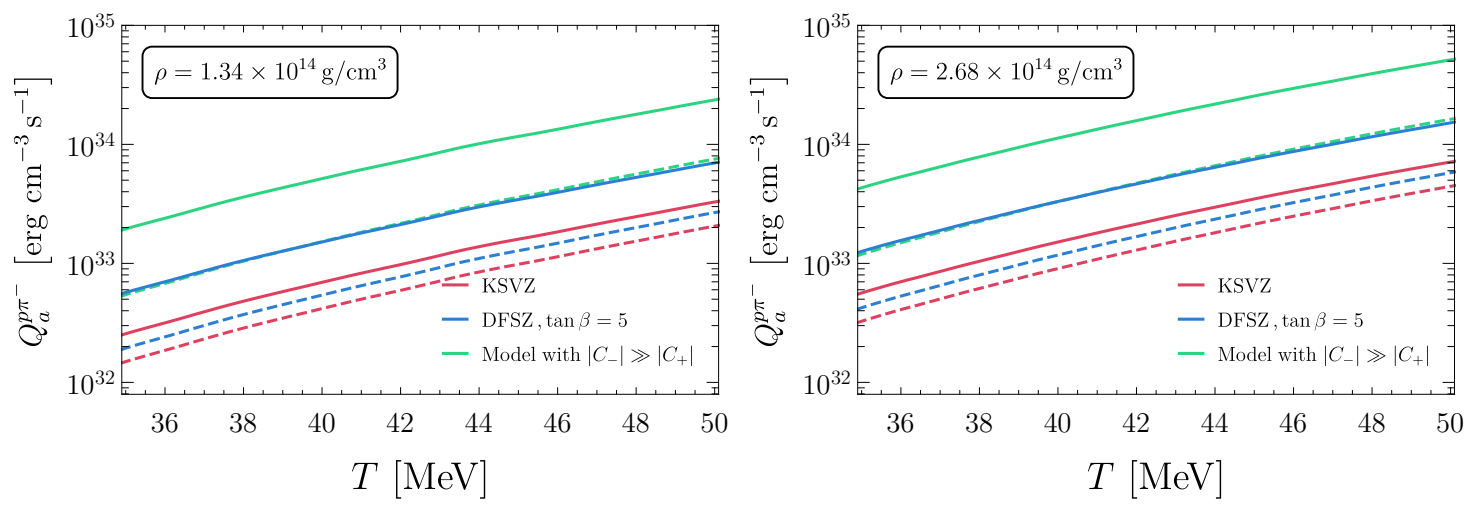

Figure 3. Axion emissivities of $\pi^{-}+p \rightarrow n+a$ for the KSVZ, DFSZ, and a model with $\left|C_{-}\right| \gg\left|C_{+}\right|$. All models are assumed to have $f_{a 9} \equiv\left(f_{a} / c_{G}\right) / 10^{9} \mathrm{GeV}=1$. The solid curves represent the total emissivity including the effect of the contact interaction $C_{a \pi N}$, while the dashed curves are the emissivity without including the contribution from $C_{a \pi N}$.

Because it is expected that the ratio is less sensitive to the uncertainty in nuclear physics, we take the ratio between each term as

$$
\frac{\Delta Q_{a, C_{a \pi N}^{2}}^{p \pi^{-}}}{\Delta Q_{a,\left(2 C_{+}^{2}+C_{-}^{2}\right)}^{p \pi^{-}}} \simeq 2.0\left(\frac{C_{-}^{2}}{2 C_{+}^{2}+C_{-}^{2}}\right), \quad \frac{\Delta Q_{a, C_{a \pi N} C_{-}}^{p \pi^{-}}}{\Delta Q_{a,\left(2 C_{+}^{2}+C_{-}^{2}\right)}^{p \pi^{-}}} \simeq 0.9\left(\frac{C_{-}^{2}}{2 C_{+}^{2}+C_{-}^{2}}\right) .
$$

Here we use the relation $C_{a \pi N}=\sqrt{2} C_{-} / g_{A}$ in eq. (2.10). Therefore, the contributions from the contact interactions enhance the axion emissivity by $\mathcal{O}(1)$ in general. We highlight in figure 3 how much $C_{a \pi N}$ enhances the axion emissivity for three benchmark axion models with $f_{a 9} \equiv\left(f_{a} / c_{G}\right) / 10^{9} \mathrm{GeV}=1$; the KSVZ model of eq. (2.7) (red), the DFSZ model of eq. (2.8) with $\tan \beta=5$ (blue), and a model (green) to realize $\left|C_{-}\right| \gg\left|C_{+}\right| \simeq 0$ by satisfying the condition eq. (2.13) for the PQ charges. For the third model, we choose $c_{G}=2$, $n_{u}=n_{d}=0, q_{H_{u}}=q_{H_{d}}=-1, \tan \beta=5$ for the model parameters in eq. (2.6), which result in $C_{a p} \simeq-C_{a n} \simeq-1.62 .{ }^{6}$ Note that in our convention, the axion decay constant $f_{a}$ is defined by the axion field range $a \cong a+2 \pi f_{a}$, and the axion-gluon coupling is given by $c_{G} / f_{a}$ for an integer-valued parameter $c_{G}$. We show that the contact interaction can enhance the axion emissivity by a factor $2-4$, depending on the pattern of axion couplings, and this conclusion will not change significantly when we include the corrections, e.g., the medium effects $[32,33]$.

Since $z_{p} / z_{n} \sim z_{\pi^{0}} / z_{\pi^{-}}=\mathcal{O}(0.1)$ inside proto-neutron star [29], the process $\pi^{0}+n \rightarrow$ $n+a$ shown in figure 2 can be as important as $\pi^{-}+p \rightarrow n+a$. Taking the same approach as eq. (3.6), the axion emissivity of $\pi^{0}+n \rightarrow n+a$ can be approximated as

$$
Q_{a}^{n \pi^{0}} \simeq \frac{1}{2} \frac{z_{n} z_{\pi^{0}}}{f_{a}^{2} f_{\pi}^{2}} \sqrt{\frac{m_{N}^{7} T^{11}}{128 \pi^{10}}} \int_{0}^{\infty} d x_{n} \frac{x_{n}^{2} e^{x_{n}^{2}}}{\left(e^{x_{n}^{2}}+z_{n}\right)^{2}} \int_{0}^{\infty} d x_{\pi}\left(\frac{x_{\pi}^{2} \epsilon_{\pi^{0}} \mathcal{C}_{a}^{n \pi^{0}}}{e^{\epsilon_{\pi^{0}}-y_{\pi^{0}}}-z_{\pi^{0}}}\right),
$$

\footnotetext{
${ }^{6} \mathrm{~A}$ simple way to realize such a case is to introduce PQ-charged exotic quarks in the minimal DFSZ model, which generate $\Delta c_{G}=-4$.
} 

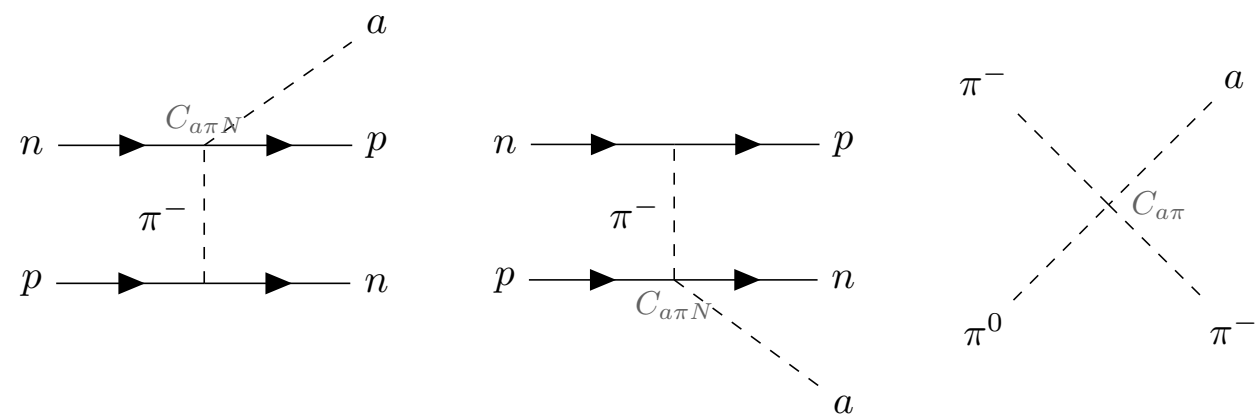

Figure 4. Other diagrams involving the contact interactions.

where $\epsilon_{\pi^{0}}=E_{\pi^{0}} / T, y_{\pi^{0}}=m_{\pi^{0}} / T$, and

$$
\mathcal{C}_{a}^{n \pi^{0}} \simeq \frac{4}{3} g_{A}^{2}\left(\frac{\left|\mathbf{p}_{\pi}\right|}{m_{N}}\right)^{2} C_{a n}^{2}
$$

Using again the fugacities obtained in [29], $Q_{a}^{n \pi^{0}}$ can be further approximated as

$$
\frac{Q_{a}^{n \pi^{0}}}{\mathrm{erg} \cdot \mathrm{cm}^{-3} \mathrm{~s}^{-1}} \simeq 1.5 \times 10^{33} T_{40}^{7.5} \rho_{14}^{1.0}\left(\frac{10^{9} \mathrm{GeV}}{f_{a}}\right)^{2} C_{a n}^{2}
$$

for $T_{40} \in[0.9,1.1]$ and $\rho_{14} \in[1,3]$. This shows that $Q_{a}^{n \pi^{0}}$ can be comparable to $Q_{a}^{p \pi^{-}}$for $T \sim 40 \mathrm{MeV}$ and $\rho \sim 10^{14} \mathrm{~g} / \mathrm{cm}^{3}$, unless $\left|C_{a n}\right| \ll\left|C_{a p}\right|$.

It is also straightforward to confirm that the other pion-nucleon scattering processes, i.e. $\pi^{0}+p \rightarrow p+a$ and $\pi^{+}+n \rightarrow p+a$, give subleading contribution relative to $\pi^{-}+p \rightarrow n+a$ and $\pi^{0}+n \rightarrow n+a$ for $T \sim 40 \mathrm{MeV}$ and $\rho \sim 10^{14} \mathrm{~g} / \mathrm{cm}^{3}$. For instance, for the process $\pi^{0}+p \rightarrow p+a$, we find

$$
\frac{Q_{a}^{p \pi^{0}}}{\operatorname{erg} \cdot \mathrm{cm}^{-3} \mathrm{~s}^{-1}} \simeq 2.7 \times 10^{32} T_{40}^{10.3} \rho_{14}^{0.56}\left(\frac{10^{9} \mathrm{GeV}}{f_{a}}\right)^{2} C_{a p}^{2} \simeq \frac{z_{p}}{z_{n}} \frac{C_{a p}^{2}}{C_{a n}^{2}} \frac{Q_{a}^{n \pi^{0}}}{\mathrm{erg} \cdot \mathrm{cm}^{-3} \mathrm{~s}^{-1}},
$$

where the fugacities of [29] are used for the last expression. This shows that for $T_{40} \in$ $[0.9,1.1]$ and $\rho_{14} \in[1,3], Q_{a}^{p \pi^{0}}<Q_{a}^{p \pi^{-}}$over the entire axion parameter space.

\subsection{Nucleon-nucleon bremsstrahlung}

For many years, the nucleon-nucleon bremsstrahlung has been considered to be the dominating process for axion emission from supernovae. Although a recent study indicates that the axion emissivity of the bremsstrahlung process is sensitive to the corrections to the one-pion exchange as well as the medium effects [28], here we do a simpler analysis ignoring these corrections since we are mainly concerned with a relative importance of the axion-pion-nucleon contact interaction $C_{a \pi N}$ compared to the other axion-nucleon interactions. In [42], the same analysis has been done for the nucleon-nucleon bremsstrahlung with the contact interaction. It shows that the contribution from the contact interaction is negligible at the squared matrix element level. In this subsection, we examine the contribution from the contact interaction to the final axion emissivity including the phase 
space integration, and confirm that it is still negligible for the environmental parameters of SN 1987A. ${ }^{7}$

Among the three possible nucleon-nucleon bremsstrahlung processes, $n+n \rightarrow n+n+a$, $n+p \rightarrow n+p+a$, and $p+p \rightarrow p+p+a$, at leading order in pion-nucleon couplings only the second process is affected by $C_{a \pi N}$ through the first two diagrams of figure 4 . The axion emissivity of the three bremsstrahlung processes is given by

$$
\begin{aligned}
Q_{a}^{I}= & \int \prod_{\substack{\alpha=N_{1}, N_{2}, N_{3}, N_{4}, a}} \frac{d^{3} \mathbf{p}_{\alpha}}{(2 \pi)^{3} 2 E_{\alpha}}\left[(2 \pi)^{4} \delta^{(4)}\left(p_{1}+p_{2}-p_{3}-p_{4}-p_{a}\right)\right. \\
& \left.\times f_{1}\left(p_{1}\right) f_{2}\left(p_{2}\right)\left(1-f_{3}\left(p_{3}\right)\right)\left(1-f_{4}\left(p_{4}\right)\right) \sum_{\text {spins }} S_{I}\left|\mathcal{M}_{I}\right|^{2} E_{a}\right] \quad(I=n n, n p, p p),
\end{aligned}
$$

where $p_{1,2}=\left(E_{1,2}, \mathbf{p}_{1,2}\right)$ and $p_{3,4}=\left(E_{3,4}, \mathbf{p}_{3,4}\right)$ denote the initial and final nucleon fourmomenta, $p_{a}=\left(E_{a}, \mathbf{p}_{a}\right)$ is the axion four-momentum, and $S_{I}$ is a symmetry factor for identical particles in the initial and final states, i.e., $S_{n n}=S_{p p}=1 / 4$ and $S_{n p}=1$. In the supernova environments, $\left|\mathbf{p}_{a}\right| \sim T \ll\left|\mathbf{p}_{N}\right| \sim \max \left[\sqrt{m_{N} T}, p_{F}\right]$ where $p_{F}$ is the nucleon Fermi momentum. Therefore we take the following approximation

$$
\mathbf{p}_{1}+\mathbf{p}_{2} \simeq \mathbf{p}_{3}+\mathbf{p}_{4}
$$

which simplifies the kinematics significantly. With this approximation and also at leading order in $1 / m_{N}$, the squared matrix elements averaged over the axion momentum direction are given by

$$
\begin{aligned}
\left\langle\sum_{\text {spins }}\left|\mathcal{M}_{n n}\right|^{2}\right\rangle= & \frac{16}{3} \frac{g_{A}^{4}}{f_{\pi}^{4}} \frac{m_{N}^{4}}{f_{a}^{2}} C_{a n}^{2}\left(\frac{|\mathbf{k}|^{4}}{\left(|\mathbf{k}|^{2}+m_{\pi}^{2}\right)^{2}}+\frac{|\mathbf{l}|^{4}}{\left(|\mathbf{l}|^{2}+m_{\pi}^{2}\right)^{2}}\right. \\
& \left.+(1-\beta) \frac{|\mathbf{k}|^{2}|\mathbf{l}|^{2}}{\left(|\mathbf{k}|^{2}+m_{\pi}^{2}\right)\left(|\mathbf{l}|^{2}+m_{\pi}^{2}\right)}\right) \\
\left\langle\sum_{\text {spins }}\left|\mathcal{M}_{p p}\right|^{2}\right\rangle= & \frac{16}{3} \frac{g_{A}^{4}}{f_{\pi}^{4}} \frac{m_{N}^{4}}{f_{a}^{2}} C_{a p}^{2}\left(\frac{|\mathbf{k}|^{4}}{\left(|\mathbf{k}|^{2}+m_{\pi}^{2}\right)^{2}}+\frac{|\mathbf{l}|^{4}}{\left(|\mathbf{l}|^{2}+m_{\pi}^{2}\right)^{2}}\right. \\
& \left.+(1-\beta) \frac{|\mathbf{k}|^{2}|\mathbf{l}|^{2}}{\left(|\mathbf{k}|^{2}+m_{\pi}^{2}\right)\left(|\mathbf{l}|^{2}+m_{\pi}^{2}\right)}\right), \\
\left\langle\sum_{\text {spins }}\left|\mathcal{M}_{n p}\right|^{2}\right\rangle= & \frac{16}{3} \frac{g_{A}^{2}}{f_{\pi}^{4}} \frac{m_{N}^{4}}{f_{a}^{2}}\left[g _ { A } ^ { 2 } \left\{\left(4 C_{+}^{2}+2 C_{-}^{2}\right) \frac{|\mathbf{k}|^{4}}{\left(|\mathbf{k}|^{2}+m_{\pi}^{2}\right)^{2}}+\left(C_{+}^{2}+C_{-}^{2}\right) \frac{|\mathbf{l}|^{4}}{\left(|\mathbf{l}|^{2}+m_{\pi}^{2}\right)^{2}}\right.\right. \\
& \left.-2\left(\left(C_{+}^{2}+C_{-}^{2}\right)-\left(3 C_{+}^{2}+C_{-}^{2}\right) \frac{\beta}{3}\right) \frac{|\mathbf{k}|^{2}|\mathbf{l}|^{2}}{\left(|\mathbf{k}|^{2}+m_{\pi}^{2}\right)\left(|\mathbf{l}|^{2}+m_{\pi}^{2}\right)}\right\} \\
& \left.+3 C_{a \pi N}^{2} \frac{\left|\mathbf{p}_{a}\right|^{2}|\mathbf{k}|^{2}}{\left(|\mathbf{k}|^{2}+m_{\pi}^{2}\right)^{2}}\right]
\end{aligned}
$$

\footnotetext{
${ }^{7}$ In appendix A, we estimate the axion emissivity in the degenerate limit by applying the analytic method presented in [27]. In that estimation, the contact interaction seems to contribute to the axion emissivity in the same order of magnitude, but the environmental parameters given in $[29,32]$ turn out to be not degenerate enough to apply the method.
} 
where

$$
\mathbf{k} \equiv \mathbf{p}_{1}-\mathbf{p}_{3}, \quad \mathbf{l} \equiv \mathbf{p}_{1}-\mathbf{p}_{4}, \quad \beta \equiv 3\left(\frac{\mathbf{k} \cdot \mathbf{l}}{|\mathbf{k}||\mathbf{l}|}\right)^{2} .
$$

For the neutron-proton bremsstrahlung, we define the momentum exchanges as $\mathbf{k}=\mathbf{p}_{n}^{i}-\mathbf{p}_{p}^{f}$ and $\mathbf{l}=\mathbf{p}_{n}^{i}-\mathbf{p}_{n}^{f}$.

While the squared matrix elements of $n+n \rightarrow n+n+a$ and $p+p \rightarrow p+p+a$ are the same as the previous results [28], the squared matrix element of $n+p \rightarrow n+p+a$ includes an additional contribution from the contact interaction $C_{a \pi N}$. We remark that we have only displayed the leading-order contribution (in $1 / m_{N}$ ) for each coupling term in the angleaveraged squared matrix elements. Then, compared to other terms, the term induced by $C_{a \pi N}$ in eq. (3.17) is intrinsically higher order as it is suppressed by $\left|\mathbf{p}_{a}\right|^{2} /\left|\mathbf{p}_{N}\right|^{2} \sim T / m_{N}$ for $|\mathbf{k}| \sim|\mathbf{l}| \sim\left|\mathbf{p}_{N}\right|$. This indicates that the contribution from $C_{a \pi N}$ to the axion emissivity of $n+p \rightarrow n+p+a$ is likely to be negligible as pointed out in [42]. If the typical values into the kinetic parameters are taken, e.g., $T=40 \mathrm{MeV}, \beta \simeq 1.3$ (non-degenerate limit), $|\mathbf{k}| \sim$ $|\mathbf{l}| \sim\left|\mathbf{p}_{N}\right| \simeq \sqrt{3 m_{N} T} \simeq 340 \mathrm{MeV},\left|\mathbf{p}_{a}\right| \simeq E_{a} \simeq\left|\mathbf{p}_{N}\right|^{2} /\left(2 m_{N}\right) \simeq 60 \mathrm{MeV}$, we could see a numerical estimate of the square brackets in eq. (3.17), $[\cdots] \simeq 6.6 C_{+}^{2}+2.2 C_{-}^{2}+0.07 C_{a \pi N}^{2}$. The estimation predicts a relative importance of each term, which is shown in eq. (3.22), although there appears some enhancement of the contribution from the contact interaction after the phase space integration.

The axion emissivity in eq. (3.13) can be simplified by taking non-relativistic limit for nucleons together with the approximation eq. (3.14). Following [25, 26], we can write the axion emissivities in a form which allows a numerical calculation of the phase space integration:

$$
\begin{aligned}
Q_{a}^{I} \simeq & \sqrt{\frac{m_{N} T^{13}}{2^{9} \pi^{16}}} \int_{0}^{\infty} d u_{+} \int_{0}^{\infty} d u_{-} \int_{-1}^{1} d \gamma_{+-} \int_{0}^{u_{-}} d u_{3 c} \int_{4 \pi} d \Omega_{3 c} \sqrt{u_{+} u_{-} u_{3 c}}\left(u_{-}-u_{3 c}\right)^{2} \\
& \times f_{1} f_{2}\left(1-f_{3}\right)\left(1-f_{4}\right) \sum_{\text {spins }} S_{I}\left\langle\left|\mathcal{M}_{I}\right|^{2}\right\rangle_{\mathbf{p}_{4 c}=-\mathbf{p}_{3 c}, E_{a}=2 T\left(u_{-}-u_{3 c}\right)}
\end{aligned}
$$

where

$$
u_{i} \equiv \frac{\mathbf{p}_{i}^{2}}{2 m_{N} T}, \quad \mathbf{p}_{ \pm} \equiv \frac{\mathbf{p}_{1} \pm \mathbf{p}_{2}}{2}, \quad \mathbf{p}_{j c} \equiv \mathbf{p}_{j}-\mathbf{p}_{+}, \quad \gamma_{k l} \equiv \frac{\mathbf{p}_{k} \cdot \mathbf{p}_{l}}{\left|\mathbf{p}_{k}\right|\left|\mathbf{p}_{l}\right|}
$$

Again, we use the fugacities of nucleons from [29] to numerically calculate the above axion 
emissivities, which results in ${ }^{8}$

$$
\begin{aligned}
\frac{Q_{a}^{n n}}{\operatorname{erg} \cdot \mathrm{cm}^{-3} \mathrm{~s}^{-1}} \simeq & 3.5 \times 10^{33} T_{40}^{3.9} \rho_{14}^{2.1}\left(\frac{10^{9} \mathrm{GeV}}{f_{a}}\right)^{2} C_{a n}^{2}, \\
\frac{Q_{a}^{n p}}{\operatorname{erg} \cdot \mathrm{cm}^{-3} \mathrm{~s}^{-1}} \simeq & 7.5 \times 10^{33} T_{40}^{6.9} \rho_{14}^{1.5}\left(\frac{10^{9} \mathrm{GeV}}{f_{a}}\right)^{2} C_{+}^{2} \\
& +2.5 \times 10^{33} T_{40}^{6.9} \rho_{14}^{1.5}\left(\frac{10^{9} \mathrm{GeV}}{f_{a}}\right)^{2} C_{-}^{2} \\
& +2.7 \times 10^{32} T_{40}^{7.9} \rho_{14}^{1.5}\left(\frac{10^{9} \mathrm{GeV}}{f_{a}}\right)^{2} C_{a \pi N}^{2}, \\
\frac{Q_{a}^{p p}}{\operatorname{erg} \cdot \mathrm{cm}^{-3} \mathrm{~s}^{-1}} \simeq & 9.9 \times 10^{31} T_{40}^{9.9} \rho_{14}^{0.92}\left(\frac{10^{9} \mathrm{GeV}}{f_{a}}\right)^{2} C_{a p}^{2}
\end{aligned}
$$

for $T_{40} \in[0.9,1.1]$ and $\rho_{14} \in[1,3]$.

\footnotetext{
${ }^{8}$ Our numerical results agree well with the analytic results of the previous works [25, 27]; for the contributions from $C_{ \pm}$, the agreement is at the level of $\mathcal{O}(10) \%$ discrepancy in both degenerate $\left(z_{n / p} \gg 1\right)$ and non-degenerate $\left(z_{n / p} \ll 1\right)$ limits. We also confirm that the contribution from $C_{a \pi N}$ agrees well with an analytic result in the degenerate limit [27]. See appendix A.
} 

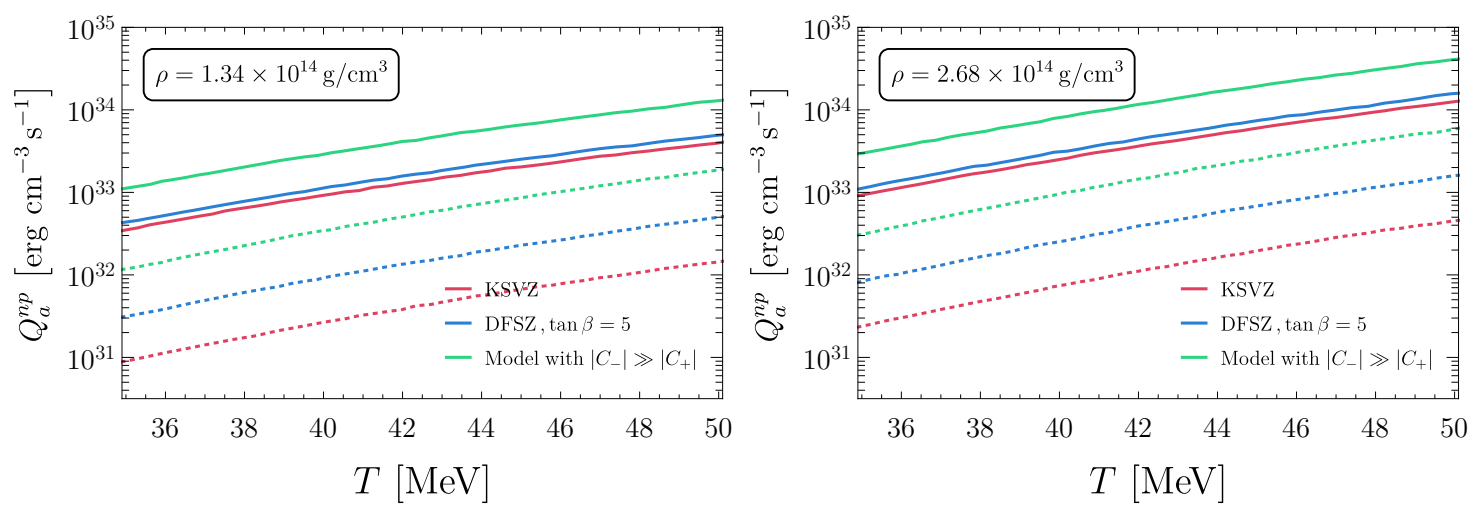

Figure 5. The axion emissivity $Q_{a}^{n p}$ for the three benchmark models considered in figure 3 . The solid (dotted) curves correspond to the total value of $Q_{a}^{n p}$ (the piece induced only by $C_{a \pi N}$ ).

The above result shows that, as anticipated from the structure of the squared matrix element, the contribution to $Q_{a}^{n p}$ from $C_{a \pi N}$ is indeed about one order of magnitude smaller than the contribution from $C_{-}$for astrophysical environments with $T \sim 40 \mathrm{MeV}$ and $\rho \sim$ $10^{14} \mathrm{~g} / \mathrm{cm}^{3}$. Using the relation $C_{a \pi N}=\sqrt{2} C_{-} / g_{A}$, the ratios between the contribution from the contact interaction and the other terms become, respectively,

$$
\frac{\Delta Q_{a, C_{a \pi N}^{2}}^{n p}}{\Delta Q_{a, C_{+}^{2}}^{n p}} \simeq 0.04\left(\frac{C_{a \pi N}^{2}}{C_{+}^{2}}\right), \quad \frac{\Delta Q_{a, C_{a \pi N}^{2}}^{n p}}{\Delta Q_{a, C_{-}^{2}}^{n p}} \simeq 0.1 .
$$

As in the case of $Q_{a}^{p \pi^{-}}$, these ratios are expected to be less sensitive to the corrections beyond the one-pion exchange and the medium effects, so the effect of the contact interaction $C_{a \pi N}$ on the nucleon-nucleon bremsstrahlung is negligible. In figure 5, we compare the total value of the axion emissivity $Q_{a}^{n p}$ (solid curves) with the piece $\Delta Q_{a, C_{a \pi N}^{2}}^{n p}$ (dotted curves) induced only by $C_{a \pi N}$ for the three benchmark models considered in figure 3 . The result shows that the contribution from $C_{a \pi N}$ is negligible for $30 \lesssim T / \mathrm{MeV} \lesssim 50$ and $1 \lesssim \rho /\left(10^{14} \mathrm{~g} / \mathrm{cm}^{3}\right) \lesssim 3$, which is expected to be true for even wider range of $T$ and $\rho$. Note that $Q_{a}^{n p}$ is comparable to (or even larger than) $Q_{a}^{n n}$, although the proton number density is significantly smaller than the neutron number density. This is partly due to the symmetry factor $S$ compensating the small proton fraction. ${ }^{9}$

\subsection{Pion-pion scattering: $\pi+\pi \rightarrow \pi+a$}

Let us finally consider the possible consequence of the axion-pion contact interaction $C_{a \pi}$ in eq. (2.9). Axions can be produced by this coupling through the pion-pion scattering process $\pi^{-}+\pi^{0} \rightarrow \pi^{-}+a$ (see the third diagram in figure 4 ). The corresponding emissivity can be simplified without any kinematic approximation as follows:

$$
\begin{aligned}
& Q_{a}^{\pi^{-} \pi^{0}}=\frac{9}{2^{12} \pi^{7}} \frac{C_{a \pi}^{2}}{f_{\pi}^{2} f_{a}^{2}} z_{\pi^{-}} z_{\pi^{0}} T^{9} \int d x_{\mathrm{in}} d x_{0} d \Omega_{\pi^{-}}^{\text {in }} d \Omega_{\pi^{0}} \frac{x_{\mathrm{in}}^{2}}{\sqrt{y_{\pi}^{2}+x_{\mathrm{in}}^{2}}} \frac{x_{0}^{2}}{\sqrt{y_{\pi}^{2}+x_{0}^{2}}} \frac{x_{a}^{2}}{E_{\mathrm{out}} / T} \\
& \times\left(\frac{\mathbf{p}_{\pi^{0}} \cdot \mathbf{p}_{a}}{T^{2}}\right)^{2} \frac{1}{e^{\sqrt{y_{\pi}^{2}+x_{\mathrm{in}}^{2}}-y_{\pi}}-z_{\pi^{-}}} \frac{1}{e^{\sqrt{y_{\pi}^{2}+x_{0}^{2}}-y_{\pi}}-z_{\pi^{0}}} \frac{e^{E_{\mathrm{out}} / T-y_{\pi}}}{e^{E_{\mathrm{out}} / T-y_{\pi}}-z_{\pi^{-}}},
\end{aligned}
$$

\footnotetext{
${ }^{9}$ The relative importance of the neutron-proton bremsstrahlung is discussed within the framework of the neutrino emission through the nucleon-nucleon bremsstrahlung [43].
} 
where $x_{\text {in }}=\left|\mathbf{p}_{\pi^{-}}^{\text {in }}\right| / T$ for the incoming $\pi^{-}, x_{0}=\left|\mathbf{p}_{\pi^{0}}\right| / T, x_{a}=\left|\mathbf{p}_{\mathbf{a}}\right| / T, y_{\pi}=m_{\pi} / T$, and finally $E_{\text {out }}=\sqrt{m_{\pi}^{2}+\left(\mathbf{p}_{\pi^{-}}^{\text {in }}+\mathbf{p}_{\pi^{0}}-\mathbf{p}_{a}\right)^{2}}$ is the energy of the outgoing $\pi^{-}$. Like the emissivity of other processes, we use the fugacities of pions from [29] and calculate the integral in eq. (3.25) numerically to find

$$
\frac{Q_{a}^{\pi^{-} \pi^{0}}}{\mathrm{erg} \cdot \mathrm{cm}^{-3} \mathrm{~s}^{-1}}=3.6 \times 10^{31} T_{40}^{10.1} \rho_{14}^{1.1}\left(\frac{10^{9} \mathrm{GeV}}{f_{a}}\right)^{2} C_{a \pi}^{2} .
$$

The above result shows that the axion emissivity of the pion-pion scattering $\pi^{-}+\pi^{0} \rightarrow$ $\pi^{-}+a$ is negligible compared to that of $\pi^{-}+p \rightarrow n+a$ for $T \sim 40 \mathrm{MeV}$ and $\rho \sim 10^{14} \mathrm{~g} / \mathrm{cm}^{3}$ and $C_{a \pi}=2\left(C_{a p}-C_{a n}\right) /\left(3 g_{A}\right)$ (see eq. $\left.(2.10)\right)$.

\section{Conclusions and discussion}

In this paper, we have studied the axion emission from supernovae with a complete set of relevant axion couplings including the axion-pion-nucleon contact interaction $C_{a \pi N}$ and the axion-pion contact interaction $C_{a \pi}$ in eq. (2.9). A recent study suggests that the abundance of negatively charged pions inside supernovae is significantly enhanced by the strong interactions [29], indicating that the pion-induced process $\pi^{-}+p \rightarrow a+n$ is the dominating process for a wide range of astrophysical conditions encountered inside supernovae [32, 33]. We thus examined how this pion-induced process is affected by $C_{a \pi N}$. We also examined the effect of $C_{a \pi N}$ on the nucleon-nucleon bremsstrahlung which has been considered as the dominating process for many years.

Since we are mainly concerned with the role of the two previously ignored couplings $C_{a \pi N}$ and $C_{a \pi}$, we have focused on the axion coupling dependence of the axion emissivity within a simple approximation to keep only the leading order in pion-nucleon couplings, which also ignores medium effects. In such an approximation, we could show the axion coupling dependence more explicitly and examine the ignored couplings for three processes. Two processes, $\pi^{-}+p \rightarrow a+n$ and $n+p \rightarrow n+p+a$, are affected by $C_{a \pi N}$, and the pion-pion scattering, $\pi+\pi \rightarrow \pi+a$, is affected by $C_{a \pi}$. We found that $C_{a \pi N}$ can enhance the axion emissivity of $\pi^{-}+p \rightarrow a+n$ by a factor of $2-4$, depending on the pattern of axion couplings determined by the underlying axion model, while there is no substantial effect on $n+p \rightarrow n+p+a$. Although it is independent of $C_{a \pi N}$, we have also examined the axion emissivity of $\pi^{0}+n \rightarrow n+a$ and find that it can be comparable to the emissivity of $\pi^{-}+p \rightarrow a+n$ over a wide range of axion parameter space. For the axion-pion contact interaction $C_{a \pi}$, we find that the corresponding axion emissivity is always negligible compared to that of $\pi^{-}+p \rightarrow a+n$ for ambient conditions encountered inside supernovae.

Let us make final remarks on the approximation we made. For the matrix elements, the higher-order diagrams could give comparable contributions due to the strong interaction. Moreover, the medium effects significantly change the axion emissivity, particularly for the nucleon-nucleon bremsstrahlung [28,33]. However, even including these effects, the relative contribution of the axion-pion-nucleon contact interaction $C_{a \pi N}$ to the axion emissivity 
would remain similar because it is likely that the ratio $\Delta Q_{a, C_{a \pi N}}^{I} / Q_{a}^{I}$ is less sensitive to the corrections than the emissivity itself, where $Q_{a}^{I}$ is the axion emissivity of the $I$-th process, and $\Delta Q_{a, C_{a \pi N}}^{I}$ is the part of $Q_{a}^{I}$ induced by $C_{a \pi N}$. One of the purposes of this work is to call attention to the possible importance of the contact interactions which have been neglected so far. It would be interesting to perform the analysis taking into account more precise matrix elements and medium effects with a complete set of axion couplings. We will investigate this issue in a self-consistent way for both the pion-nucleon scattering and the nucleon-nucleon bremsstrahlung in future works.

\section{Acknowledgments}

This work was supported by IBS under the project code, IBS-R018-D1. We are grateful to S. Yun for helpful discussions, especially on the nucleon-nucleon bremsstrahlung process in the early stage of this project.

\section{A Axion emissivity from $n+p \rightarrow n+p+a$ in degenerate limit}

In the degenerate regime, the axion emissivity for the nucleon-nucleon bremsstrahlung can be derived in an analytical way because the phase space is highly constrained [27]. We can apply the method to include the contribution from the contact term, which is a higher order in the expansion with respect to $\left|\mathbf{p}_{a}\right| /\left|\mathbf{p}_{N}\right| \sim \sqrt{T / m_{N}}$ (see eq. (3.17)). Although our system belongs to the non-degenerate regime, the analytical estimation provides some insights for the numerical results. Moreover, the analytical result is applicable for the degenerate system like a neutron star.

$$
\begin{aligned}
\frac{\left(\Delta Q_{a}^{n p}\right)_{C_{a \pi N}^{2}}}{\left(Q_{a}^{n p}\right)_{C_{a \pi N}=0}}= & \frac{123 \pi^{2}}{124 g_{A}^{2}} \frac{T^{2}}{p_{F, n}^{2}} \frac{y}{x^{2}} C_{a \pi N}^{2}\left[H\left(\frac{2 x y}{x+y}\right)-H\left(\frac{2 x y}{y-x}\right)\right] \\
& \times\left[\left(C_{-}^{2}+C_{+}^{2}\right) F(y)-2\left(C_{-}^{2}+C_{+}^{2}\right) G(y)\right. \\
& \left.+\left(C_{-}^{2}+2 C_{+}^{2}\right)\left\{\left(1-\frac{y}{x}\right) F\left(\frac{2 x y}{y-x}\right)+\left(1+\frac{y}{x}\right) F\left(\frac{2 x y}{x+y}\right)\right\}\right]^{-1}, \\
\simeq & \frac{C_{a \pi N}^{2}}{C_{-}^{2}+3 C_{+}^{2}}\left(\frac{7.0 T}{p_{F, n}}\right)^{2}, \\
= & \frac{\left(C_{a p}-C_{a n}\right)^{2}}{\left(C_{a p}-C_{a n}\right)^{2}+3\left(C_{a p}+C_{a n}\right)^{2}}\left(\frac{7.7 T}{p_{F, n}}\right)^{2},
\end{aligned}
$$

where $x \equiv m_{\pi} /\left(2 p_{F, n}\right), y \equiv m_{\pi} /\left(2 p_{F, p}\right)$, and

$$
\begin{aligned}
F(u) & \equiv \frac{1}{2}\left(3-\frac{1}{1+u^{2}}-3 u \cot ^{-1} u\right), \\
G(v) & \equiv 1-v \cot ^{-1} v, \\
H(w) & \equiv-\frac{w}{1+w^{2}}+\cot ^{-1} w .
\end{aligned}
$$




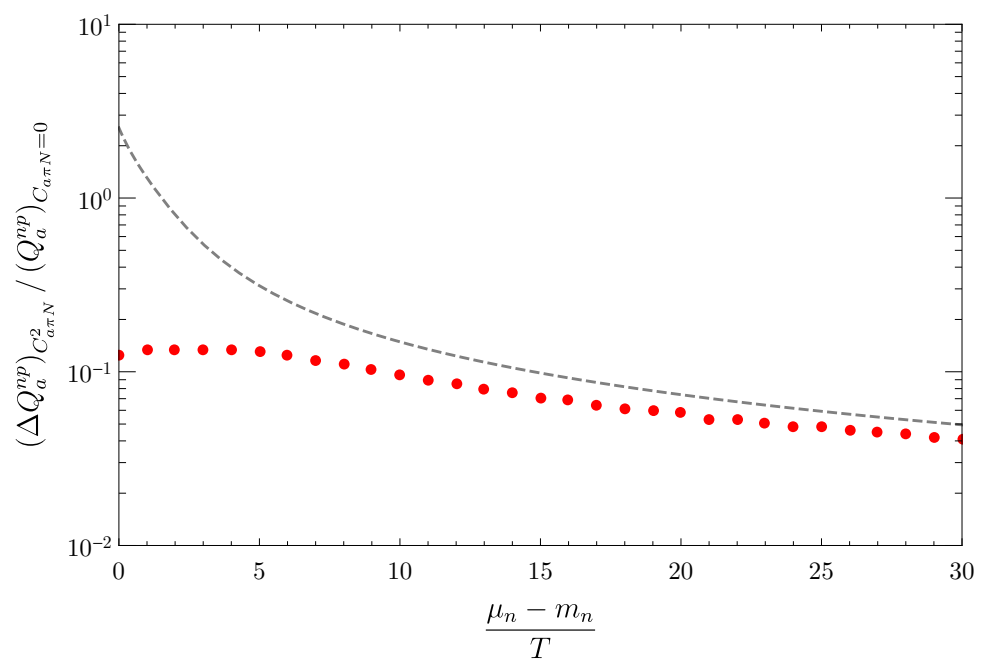

Figure 6. The ratio of the axion emissivity contributed from the contact interaction, i.e. $C_{a \pi N}$ coupling, to that from the other couplings, $C_{a p}$ and $C_{a n}$. We compare the numerical result (red dots) with an analytic estimation (dashed). For benchmark parameters, we assume $\mu_{p}-m_{p} \simeq$ $0.25\left(\mu_{n}-m_{n}\right)$ and $C_{a p}=-C_{a n}=1 / 2$.

For the second equality of eq. (A.1), we keep the leading order with respect to $x$ and $y$. Given the parameter values in the main text, e.g. $T \simeq 40 \mathrm{MeV}$ and $p_{F, n} \simeq 200-300 \mathrm{MeV}$, eq. (A.1) leads to the wrong interpretation that the new contribution from the contact interaction is comparable to the other contributions from the $C_{a p}$ and $C_{a n}$ couplings. The discrepancy between the analytic estimation in eq. (A.1) and the numerical results in eq. (3.22) is caused by applying the formula derived in the degenerate limit to the nondegenerate system [32]. Figure 6 shows the emissivity ratio of the $C_{a \pi N}^{2}$ contribution with respect to the other contributions from the axion-nucleon couplings for $\mu_{n}>m_{n}$. Actually, the chemical potential for the proton in the supernova environments [29] corresponds to the case of $\mu_{p}<m_{p}$. In order to highlight the difference between the emissivity calculated in degenerated limit and that for non-degenerate case, the proton chemical potential is taken to have a simple relation as $\mu_{p}-m_{p} \simeq 0.25\left(\mu_{n}-m_{n}\right)$ and we choose $C_{a p}=-C_{a n}=1 / 2$. The analytic estimation for the emissivity ratio is shown in figure 6 as a dashed line. Figure 6 gives information on the validity of the degenerate limit.

Open Access. This article is distributed under the terms of the Creative Commons Attribution License (CC-BY 4.0), which permits any use, distribution and reproduction in any medium, provided the original author(s) and source are credited.

\section{References}

[1] R.D. Peccei and H.R. Quinn, CP conservation in the presence of instantons, Phys. Rev. Lett. 38 (1977) 1440 [INSPIRE].

[2] R.D. Peccei and H.R. Quinn, Constraints imposed by CP conservation in the presence of instantons, Phys. Rev. D 16 (1977) 1791 [INSPIRE]. 
[3] S. Weinberg, A new light boson?, Phys. Rev. Lett. 40 (1978) 223 [inSPIRE].

[4] F. Wilczek, Problem of strong $P$ and $T$ invariance in the presence of instantons, Phys. Rev. Lett. 40 (1978) 279 [INSPIRE].

[5] J.E. Kim and G. Carosi, Axions and the Strong CP Problem, Rev. Mod. Phys. 82 (2010) 557 [Erratum ibid. 91 (2019) 049902] [arXiv:0807.3125] [INSPIRE].

[6] L. Di Luzio, M. Giannotti, E. Nardi and L. Visinelli, The landscape of QCD axion models, Phys. Rept. 870 (2020) 1 [arXiv: 2003. 01100] [INSPIRE].

[7] K. Choi, S.H. Im and C. Sub Shin, Recent progress in the physics of axions and axion-like particles, Ann. Rev. Nucl. Part. Sci. 71 (2021) 225 [arXiv: 2012.05029] [InSPIRE].

[8] J. Preskill, M.B. Wise and F. Wilczek, Cosmology of the invisible axion, Phys. Lett. B 120 (1983) 127 [INSPIRE].

[9] L.F. Abbott and P. Sikivie, A cosmological bound on the invisible axion, Phys. Lett. B 120 (1983) 133 [INSPIRE].

[10] M. Dine and W. Fischler, The not so harmless axion, Phys. Lett. B 120 (1983) 137 [INSPIRE].

[11] J.E. Kim, Weak interaction singlet and strong CP invariance, Phys. Rev. Lett. 43 (1979) 103 [INSPIRE].

[12] M.A. Shifman, A.I. Vainshtein and V.I. Zakharov, Can confinement ensure natural CP invariance of strong interactions?, Nucl. Phys. B 166 (1980) 493 [INSPIRE].

[13] M. Dine, W. Fischler and M. Srednicki, A simple solution to the strong CP problem with a harmless axion, Phys. Lett. B 104 (1981) 199 [INSPIRE].

[14] A.R. Zhitnitsky, On possible suppression of the axion hadron interactions (in Russian), Sov. J. Nucl. Phys. 31 (1980) 260 [Yad. Fiz. 31 (1980) 497] [inSPIRE].

[15] P.W. Graham, I.G. Irastorza, S.K. Lamoreaux, A. Lindner and K.A. van Bibber, Experimental searches for the axion and axion-like particles, Ann. Rev. Nucl. Part. Sci. 65 (2015) 485 [arXiv : 1602.00039] [INSPIRE].

[16] I.G. Irastorza and J. Redondo, New experimental approaches in the search for axion-like particles, Prog. Part. Nucl. Phys. 102 (2018) 89 [arXiv:1801.08127] [InSPIRE].

[17] Y.K. Semertzidis and S. Youn, Axion dark matter: how to see it?, arXiv:2104.14831 [INSPIRE].

[18] G.G. Raffelt, Astrophysical axion bounds, Lect. Notes Phys. 741 (2008) 51 [hep-ph/0611350] [INSPIRE].

[19] L. Di Luzio, M. Fedele, M. Giannotti, F. Mescia and E. Nardi, Stellar evolution confronts axion models, arXiv:2109.10368 [INSPIRE].

[20] M.S. Turner, Axions from SN 1987a, Phys. Rev. Lett. 60 (1988) 1797 [InSPIRE].

[21] G. Raffelt and D. Seckel, Bounds on exotic particle interactions from SN 1987a, Phys. Rev. Lett. 60 (1988) 1793 [INSPIRE].

[22] A. Burrows, Supernova explosions in the universe, Nature 403 (2000) 727 [INSPIRE].

[23] S. Woosley and T. Janka, The physics of core-collapse supernovae, Nature Phys. 1 (2005) 147 [astro-ph/0601261] [INSPIRE].

[24] N. Iwamoto, Axion emission from neutron stars, Phys. Rev. Lett. 53 (1984) 1198 [INSPIRE]. 
[25] R.P. Brinkmann and M.S. Turner, Numerical rates for nucleon-nucleon axion bremsstrahlung, Phys. Rev. D 38 (1988) 2338 [inSPIRE].

[26] G. Raffelt and D. Seckel, A selfconsistent approach to neutral current processes in supernova cores, Phys. Rev. D 52 (1995) 1780 [astro-ph/9312019] [InSPIRE].

[27] N. Iwamoto, Nucleon-nucleon bremsstrahlung of axions and pseudoscalar particles from neutron star matter, Phys. Rev. D 64 (2001) 043002 [INSPIRE].

[28] P. Carenza, T. Fischer, M. Giannotti, G. Guo, G. Martínez-Pinedo and A. Mirizzi, Improved axion emissivity from a supernova via nucleon-nucleon bremsstrahlung, JCAP 10 (2019) 016 [Erratum ibid. 05 (2020) E01] [arXiv:1906.11844] [INSPIRE].

[29] B. Fore and S. Reddy, Pions in hot dense matter and their astrophysical implications, Phys. Rev. C 101 (2020) 035809 [arXiv: 1911.02632] [inSPIRE].

[30] M.S. Turner, Dirac neutrinos and SN1987A, Phys. Rev. D 45 (1992) 1066 [InSPIRE].

[31] W. Keil, H.-T. Janka, D.N. Schramm, G. Sigl, M.S. Turner and J.R. Ellis, A fresh look at axions and SN-1987A, Phys. Rev. D 56 (1997) 2419 [astro-ph/9612222] [INSPIRE].

[32] P. Carenza, B. Fore, M. Giannotti, A. Mirizzi and S. Reddy, Enhanced supernova axion emission and its implications, Phys. Rev. Lett. 126 (2021) 071102 [arXiv:2010.02943] [INSPIRE].

[33] T. Fischer, P. Carenza, B. Fore, M. Giannotti, A. Mirizzi and S. Reddy, Observable signatures of enhanced axion emission from protoneutron stars, Phys. Rev. D 104 (2021) 103012 [arXiv:2108.13726] [INSPIRE].

[34] R. Balkin, J. Serra, K. Springmann and A. Weiler, The QCD axion at finite density, JHEP 07 (2020) 221 [arXiv: 2003.04903] [INSPIRE].

[35] H. Georgi, D.B. Kaplan and L. Randall, Manifesting the invisible axion at low-energies, Phys. Lett. B 169 (1986) 73 [INSPIRE].

[36] K. Choi, S.H. Im, H.J. Kim and H. Seong, Precision axion physics with running axion couplings, JHEP 08 (2021) 058 [arXiv:2106.05816] [INSPIRE].

[37] Y. Ema, K. Hamaguchi, T. Moroi and K. Nakayama, Flaxion: a minimal extension to solve puzzles in the standard model, JHEP 01 (2017) 096 [arXiv: 1612.05492] [INSPIRE].

[38] L. Calibbi, F. Goertz, D. Redigolo, R. Ziegler and J. Zupan, Minimal axion model from flavor, Phys. Rev. D 95 (2017) 095009 [arXiv: 1612.08040] [INSPIRE].

[39] F. Björkeroth, E.J. Chun and S.F. King, Accidental Peccei-Quinn symmetry from discrete flavour symmetry and Pati-Salam, Phys. Lett. B 777 (2018) 428 [arXiv:1711.05741] [INSPIRE].

[40] S. Chang and K. Choi, Hadronic axion window and the big bang nucleosynthesis, Phys. Lett. B 316 (1993) 51 [hep-ph/9306216] [INSPIRE].

[41] G. Grilli di Cortona, E. Hardy, J. Pardo Vega and G. Villadoro, The QCD axion, precisely, JHEP 01 (2016) 034 [arXiv:1511.02867] [INSPIRE].

[42] M. Carena and R.D. Peccei, The effective lagrangian for axion emission from SN1987A, Phys. Rev. D 40 (1989) 652 [inSPIRE].

[43] D.G. Yakovlev, A.D. Kaminker, O.Y. Gnedin and P. Haensel, Neutrino emission from neutron stars, Phys. Rept. 354 (2001) 1 [astro-ph/0012122] [INSPIRE]. 